\title{
Coherent effects in double-barrier Josephson junctions
}

\author{
A. Brinkman, A. A. Golubov \\ Department of Applied Physics, University of Twente, \\ P.O.Box 217, 7500 AE Enschede, The Netherlands
}

(August 13, 2018)

\begin{abstract}
The general solution for ballistic electronic transport through double-barrier Josephson junctions is derived. We show the existence of a regime of phase-coherent transport in which the supercurrent is proportional to the single barrier transparency and the way in which this coherence is destroyed for increasing interlayer thickness. The quasiparticle dc current at arbitrary voltage is determined.
\end{abstract}

PACS numbers: 74.50.+r, 74.80.Dm, 74.80.Fp

Phase-coherent electronic transport in mesoscopic structures between normal $(\mathrm{N})$ and superconducting $(\mathrm{S})$ metals receive considerable interest both in experiments and in theoryt. Particular interesting phenomena were discovered in structures containing tunnel barriers (I). It is well known that the subgap resistance of a ballistic SIN junction has a quadratic dependence on the transparency of the interface 2 , since Andreev reflection is a two-particle process. Disorder in the normal region enhances the Andreev current due to opening of some fraction of tunneling channels, and the resistance has a linear dependence on the transparency. This effect is $\mathrm{known}^{\mathrm{k}}$ reflectionless tunpeling in SIN junctions (see 3 , t and further references inl). Interestingly, the opening of tunnel channels may be realized in a ballistic $N I_{1} N I_{2} S$ junction as well by placing a second tunnel barriert.

At the same time, a supercurrent $I_{c}$ in a tunnel $S I S$ contact depends linearly on the barrier transparency since Cooper pairs tunnel coherently 6 - Here we address the problem of universal features of supercurrent flow in a double-barrier ballistic $S I_{1} S^{\prime} I_{2} S$ junction, where $S^{\prime}$ is a thin layer with critical temperature $T_{c s^{\prime}}<T_{c s}$. Coherent effects in such structures are also of practical importance, since recent experiments demonstrated the possibility of engineering Josephson junctions with desired properties using existing multilayered techniques 8,9 .

The supercurrent in a disordered double-barrier SINIS junction was calculated by Kupriyanov and Lukichev10, who considered the interlayer in the dirty limit and $T_{c s^{\prime}}=0$. The coherent regime was found in the limit of small interlayer thickness $d$, when supercurrent is of the first order in the single barrier transmission coefficient $D$, like in a $S I S$ junction. However in the limit of small $d$ the assumption of the dirty limit is not justified since electronic scattering takes place only at the interfaces. Theoretical work on ballistic SINIS structures was concentrated on studying resonant supercurrents in low-dimensional structures11 16.

In the present paper we study theoretically the universal features of charge transport in a three-dimensional (3D) $S I_{1} S^{\prime} I_{2} S$ junction in the clean limit. We demonstrate the existence of the coherent regime when the supercurrent is averaged over the transmission resonances and is proportional to $D$, whereas it becomes incoherent, of the order of $D^{2}$, with increasing thickness, as expected for two uncorrelated sequential tunneling processes. We study quantitatively the crossover between these two regimes and the relation to the dirty limit results of Ref.10. Further, we show that the coherent supercurrent can be exactly derived from the distribution of transmission eigenvalues $\rho(D) \propto D^{-3 / 2}(1-D)^{-1 / 2}$ known for a two-barrier $N I_{1} N I_{2} N$ contact 1 . Based on this distribution, we calculate the quasiparticle $d c$ current at arbitrary voltage, which shows signatures of multiple Andreev reflections (MAR).

We consider a $3 \mathrm{D}$ ballistic $S I_{1} S^{\prime} I_{2} S$ contact, where S' is a thin superconducting film with $T_{c s^{\prime}}<T_{c s}$ and the mean free path $l_{s^{\prime}}>d, d$ is the interlayer thickness and $I_{1,2}$ are the parallel atomically sharp interfaces with arbitrary transmission coefficients. In the temperature Green's function method the supercurrent density $J_{s}$ is expressed through the Fourier transform of the Green's function $G\left(\mathbf{r}, \mathbf{r}^{\prime}\right)$ over the transverse coordinates

$$
\begin{aligned}
J_{S}(x)= & \frac{i \hbar e}{m} \int \frac{d^{2} k_{\|}}{(2 \pi)^{2}} T \sum_{\omega_{n}>0} \\
& \lim _{x^{\prime} \rightarrow x}\left(\frac{\partial}{\partial x^{\prime}}-\frac{\partial}{\partial x}\right) G\left(x, x^{\prime} ; k_{\|}, \omega_{n}\right),
\end{aligned}
$$

where $x, x^{\prime}$ are the coordinates across the junction, $k_{\|}$is the wave-vector component in the junction plane, $\omega_{n}=$ $(2 n+1) \pi T$. The normal and the anomalous Green's functions $G\left(x, x^{\prime}\right), F^{+}\left(x, x^{\prime}\right)$ obey the Gor'kov equations

$$
\left(\begin{array}{cc}
i \omega_{n}+H & \widetilde{\Delta}(x) \\
\widetilde{\Delta}^{*}(x) & i \omega_{n}-H
\end{array}\right) \cdot\left(\begin{array}{c}
G \\
F^{+}
\end{array}\right)=\left(\begin{array}{c}
\delta\left(x-x^{\prime}\right) \\
0
\end{array}\right),
$$

where $\widetilde{\Delta}=\Delta \exp (i \chi)$ is the spatially dependent complex pair potential, $H=\frac{\hbar^{2}}{2 m} \frac{\partial^{2}}{\partial x^{2}}+E_{x}-V(x), E_{x}=$ $E_{F}-\hbar^{2} k_{\|}^{2} / 2 m$ is the electron kinetic energy across the junction, $E_{F}$ is the Fermi energy, $V(x)=W_{1} \delta(x)+$ $W_{2} \delta(x-d)$ is the interface potential, $W_{1,2}$ being the barrier strengths.

Let us choose the position $x^{\prime}$ within the interlayer, then the solution of Eq. (2) in the superconducting electrodes $(|x|>d / 2)$ is given by a linear combination of plane waves $A\left(x^{\prime}\right) \exp \left(i k_{x} x\right)$. Substituting it into Eq. (2), we arrive the dispersion relation for $k$ which yields four solutions: $k= \pm \sqrt{k_{F}^{2}-k_{\|}^{2}+2 i m E / \hbar^{2}}, k^{*}=$ 
$\pm \sqrt{k_{F}^{2}-k_{\|}^{2}-2 i m E / \hbar^{2}}$ with $E=\sqrt{\omega_{n}^{2}+\Delta^{2}}$ and $k_{F}^{2}=$ $2 m E_{F} / \hbar^{2}$. As a result the homogeneous solutions of Eq. (2) at $x<-d / 2$ has the form

$$
\left(\begin{array}{c}
G \\
F^{+}
\end{array}\right)=a_{ \pm}\left(\begin{array}{c}
1 \\
\beta
\end{array}\right) e^{-i k\left(x \pm x^{\prime}\right)}+b_{ \pm}\left(\begin{array}{c}
-\beta^{*} \\
1
\end{array}\right) e^{i k^{*}\left(x \pm x^{\prime}\right)}
$$

where $\beta=i \Delta^{*} /\left(\omega_{n}+E\right)$. All four terms in Eq. (3) decay properly at $x=-\infty$. A similar solution holds for $x>-d / 2$

$$
\left(\begin{array}{c}
G \\
F^{+}
\end{array}\right)=c_{ \pm}\left(\begin{array}{c}
1 \\
\beta
\end{array}\right) e^{i k\left(x \pm x^{\prime}\right)}+d_{ \pm}\left(\begin{array}{c}
-\beta^{*} \\
1
\end{array}\right) e^{-i k^{*}\left(x \pm x^{\prime}\right)} .
$$

In the case of $T_{c s^{\prime}}=0$ the solution in the interlayer $|x|<d / 2$ consists of the homogeneous part, in which $\beta=$ 0 and all terms of Eqs. (3, 4) are present, and the particular solution (source term): $G=\left(m / i \hbar^{2} k\right) e^{i k\left|x-x^{\prime}\right|}$, $F=0$.

The solutions in all three regions are matched by the conditions of continuity of the $G\left(x, x^{\prime}\right)$ and $F\left(x, x^{\prime}\right)$ at the interfaces $x= \pm d / 2$ and by the condition for the derivatives which follows from the integration of Eq. (2) across the interface barriers. This yields for $x=-d / 2$

$$
\begin{aligned}
& 0=\frac{1}{2 m_{S^{\prime}}} \frac{\partial G}{\partial x}\left(\begin{array}{c}
G \\
F^{+}
\end{array}\right)_{x=d / 2+0}-W_{1}\left(\begin{array}{c}
G \\
F^{+}
\end{array}\right)_{x=0}, \\
& -\frac{1}{2 m_{S}} \frac{\partial}{\partial x}\left(\begin{array}{c}
G \\
F^{+}
\end{array}\right)_{x=d / 2-0}-
\end{aligned}
$$

and similar condition for $x=d / 2$. These boundary conditions provide the required number of linear equations for the coefficients in the equations for $G\left(x, x^{\prime}\right)$ and $F\left(x, x^{\prime}\right)$.

We can expand the wavevector in the interlayer as

$$
k_{s^{\prime}}=\sqrt{k_{x}^{2}+2 i m \sqrt{\omega_{n}^{2}+\left|\Delta_{s^{\prime}}\right|^{2}} / \hbar^{2}} \simeq k_{x}+\frac{i}{2 \xi_{s^{\prime} x}},
$$

where $k_{x}=\sqrt{k_{F}^{2}-k_{\|}^{2}}=k_{F} \cos \theta$ is the transverse component of the wave vector, $\xi_{s^{\prime} x}=\xi_{s^{\prime}} / \cos \theta, \xi_{s^{\prime}}=$ $\hbar v_{F} / 2 \cos \theta \sqrt{\omega_{n}^{2}+\Delta_{s^{\prime}}^{2}}$ is the coherence length and $\Delta_{s^{\prime}}$ is the pair potential in the $S^{\prime}$ layer. In the $S$ electrodes we still keep $k_{S}=k_{x}$ with accuracy up to terms of the order of $\Delta / E_{F}$. By matching the solutions (3, 4) in all three regions at the interfaces we derived an expression for the supercurrent $J_{s}$, valid for arbitrary $d$ and $W_{1,2}$. Below we present the solution for $d<\xi_{s^{\prime}}$ and symmetric low-transparent barriers $W_{1,2}=W,\left(W / \hbar v_{F}\right) \gg 1$

$$
\begin{aligned}
J_{s}= & \frac{e}{\hbar} \int \frac{d^{2} k_{\|}}{(2 \pi)^{2}} T \sum_{\omega \geq 0} \\
& \frac{\Delta_{s}^{2} \sin \varphi+\Delta_{s} \Delta_{s^{\prime}} \sqrt{E_{1} / E_{2}} d / \xi_{s^{\prime}} \widetilde{W^{2}} \sin \frac{\varphi}{2}}{2 \widetilde{W^{4}} E_{1}^{2}\left(\cosh d / \xi_{s^{\prime} x}-\cos 2 k_{x} d\right)+E_{3}^{2}}
\end{aligned}
$$

Here $\varphi$ is the phase difference across the junction, $\Delta_{s}$ is the pair potential in $S, E_{1}=\sqrt{\omega_{n}^{2}+\Delta_{s}^{2}}, E_{2}=$ $\sqrt{\omega_{n}^{2}+\Delta_{s^{\prime}}^{2}}, E_{3}=\sqrt{\omega_{n}^{2}+\Delta_{s}^{2} \cos ^{2} \varphi / 2}$ and $\widetilde{W}=W / \hbar v_{F}$.

Eq. (8) is the main technical result of this paper and describes the interplay between quasiparticle transmission (Breit-Wigner) resonances and electron-hole (Andreev) resonances. Changing the phase space in integration over $k_{\|}$, one can apply Eq.(8) to the problem of the supercurrent flow via transmission resonances in low-dimensional contacts. In this case the results of 12 14.11, t5, taken in relevant limits, are reproduced. Below we concentrate on the interplay between coherent and incoherent regimes in the case of large $k_{F} d$. It is instructive to discuss this crossover in terms of the width of transmission resonances in a double-barrier junction, which in the symmetrical case is given by $\Gamma=\hbar v_{F}\langle x D(x)\rangle / 2 d=\hbar v_{F} / 8 d \widetilde{W}^{2}$, where $\langle x D(x)\rangle$ is the angle-averaged transparency of a single barrier $(x=\cos \theta)$.

Coherent regime (broad resonances). As follows from Eq.(8), the coherent regime takes place for a thin interlayer when the transmission resonances are broad $\Gamma \gg$ $\pi T_{c s}$. The supercurrent is then given by

$$
J_{S}=\frac{e}{\hbar} \int \frac{d^{2} k_{\|}}{(2 \pi)^{2}} T \sum_{\omega} \frac{\Delta_{s}^{2} \sin \varphi}{E_{1}^{2} D_{2}^{-1}-\Delta_{s}^{2} \sin ^{2} \varphi / 2},
$$

where $D_{2}$ is the transparency of a double barrier NININ contact

$$
D_{2}^{-1}=1+\left(2 \widetilde{W} \cos k_{F}^{\prime} d+2 \widetilde{W}^{2} \sin k_{F}^{\prime} d\right)^{2}
$$

and has a resonant structure. Integration over the directions of $k_{\|}$(over the resonances) yields the supercurrent

$$
e J_{s} R_{N}=2 \pi T \sum_{\omega} \frac{\Delta_{s}^{2} \sin \varphi}{E_{1} E_{3}}
$$

which does not depend on the properties of the inter layer and coincides with the dirty-limit KL result 10 . Here $R_{N}^{-1}=e^{2} k_{F}^{2} \gamma / 4 \pi^{2} \hbar$ is the normal state contact resistance per square, where $\gamma=\langle x D(x)\rangle=1 / 4 \widetilde{W}^{2}$. This expression can be generalized to the asymmetric case: $e J_{s} R_{N}=2 \pi T \sum_{\omega}\left|\Delta_{S}\right|^{2} \sin \varphi / E_{1} E_{3}^{\prime}$, with $E_{3}^{\prime}=\sqrt{\omega_{n}^{2}+\left|\Delta_{s}\right|^{2}\left(\cos ^{2} \varphi / 2+\gamma_{-}^{2} \sin ^{2} \varphi / 2\right)}, \gamma_{-}=\left(\gamma_{1}-\right.$ $\left.\gamma_{2}\right) /\left(\gamma_{1}+\gamma_{2}\right), \gamma_{1,2}=\left\langle x D_{1,2}(x)\right\rangle$, where $D_{1,2}$ are the individual barrier transparencies. In this case, $R_{N}^{-1}=$ $e^{2} k_{F}^{2} \gamma_{c} / 2 \pi^{2} \hbar$, where $\gamma_{c}=\gamma_{1} \gamma_{2} /\left(\gamma_{1}+\gamma_{2}\right)$ which is the classical result known from 17 .

Expression (11) has been found by Kupriyanov and Lukichev10 in the case of a double-barrier junction with a dirty metal interlayer in the limit of vanishingly small thickness. This fact shows that Eq. (11) is a very general result.

For $T=0$ the maximum value of $e I_{s} R_{N}$ is achieved at $\varphi \approx 1.86$ and exceeds the $e J_{c} R_{N}$ value of $(\pi / 2)\left|\Delta_{s}\right|$ for 


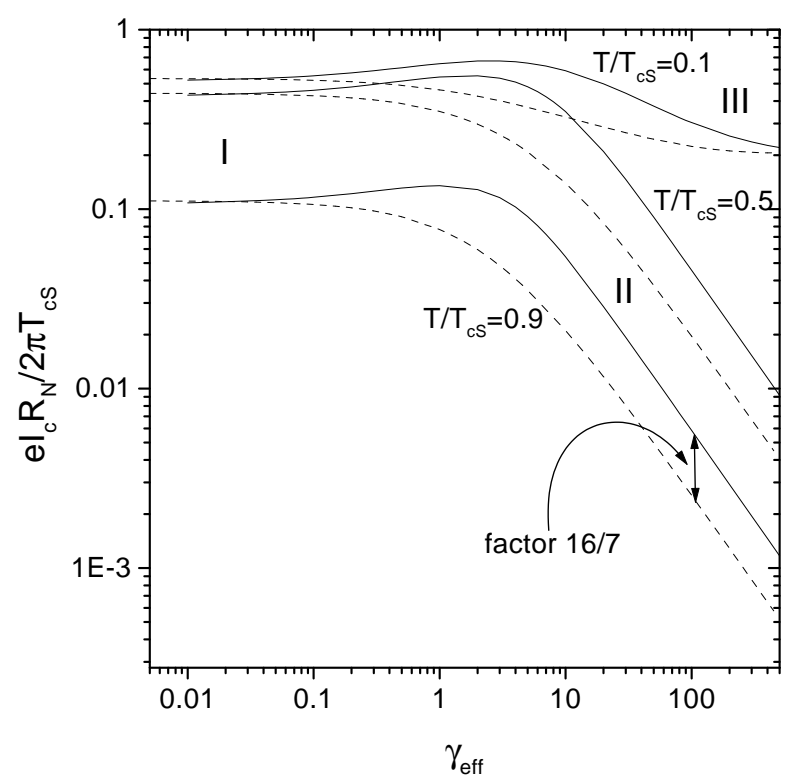

FIG. 1. $I_{c} R_{N}$ product in the model of clean interlayer (solid lines) and disordered interlayer (dashes lines). The curves are plotted for the $\mathrm{NB} / \mathrm{Al}$ case for $T_{c s}=7.4 T_{c s^{\prime}}$.

a tunnel SIS contact. The reason is that in the coherent regime the dominant contribution to $J_{s}$ comes from the transmission resonances, which in the present case are broader than $\Delta$. As a result the supercurrent is of the first order in $\langle x D(x)\rangle$.

The supercurrent in the coherent regime has the spectral density

$$
\operatorname{Im} J_{s}(\varepsilon)=\frac{\Delta_{s}^{2} \sin \varphi}{\sqrt{\Delta_{s}^{2}-\varepsilon^{2}} \sqrt{\varepsilon^{2}-\Delta_{s}^{2} \cos ^{2} \varphi / 2}},
$$

for $\Delta_{s} \cos \varphi / 2<\varepsilon<\Delta_{s}$, while $\operatorname{Im} J_{S}(\varepsilon)=0$ for $\varepsilon<$ $\Delta_{s} \cos \varphi / 2$ and $\varepsilon>\Delta_{s}$, i.e. the Andreev bound states in the energy range $\Delta_{s} \cos \varphi / 2<\varepsilon<\Delta_{s}$ contribute to the supercurrent.

Incoherent regime (narrow resonances) $\Gamma \ll \pi T_{c s}$. With the increase of the interlayer thickness the coherent regime breaks down due to the dephasing of the transmission resonances. After performing the angle averaging in Eq.(8) the general expression for a double-barrier junction becomes

$$
\begin{aligned}
e J_{s} R_{N}= & 2 \pi T \sum_{\omega_{n}} \frac{\Delta_{s}^{2} \sin \varphi}{E_{1}^{2}} \int_{0}^{1} \frac{4 x^{5} d x}{\widehat{W}^{2} \sqrt{a^{2}-1}} \\
& +\frac{\Delta_{s} \Delta_{s^{\prime}} \sin \frac{\varphi}{2}}{E_{1} E_{2}} \frac{d}{\xi_{s^{\prime}}} \int_{0}^{1} \frac{8 x^{2} d x}{\sqrt{a^{2}-1}}
\end{aligned}
$$

where $a=\cosh \left(d / \xi_{s^{\prime}} x\right)+\frac{1}{2 \widetilde{W}^{4}}\left(\Delta_{s}^{2} \cos ^{2} \frac{\varphi}{2}+\omega_{n}^{2}\right) /\left(\Delta_{s}^{2}+\omega_{n}^{2}\right)$. The pair potential in $\mathrm{S}^{\prime}$ is determined self-consistently with

$$
-\Delta_{s^{\prime}} \ln \frac{T}{T_{c s^{\prime}}}=2 \pi T \sum_{\omega_{n}}\left(\frac{\Delta_{s^{\prime}}}{\omega_{n}}-\left\langle F_{s^{\prime}}\right\rangle\right),
$$

where $\left\langle F_{S^{\prime}}\right\rangle$ is the angle-averaged anomalous Green's function, that is solved in the same way as the normal Green's function G.

$$
\left\langle F_{s^{\prime}}\right\rangle=4 \int_{0}^{1}\left(\frac{\Delta_{s^{\prime}}}{E_{2}} \sinh \left(\frac{d}{\xi_{s^{\prime}} x}\right)+\frac{2 \Delta_{s}}{E_{1}} x^{4} \cos \frac{\varphi}{2}\right) \frac{x d x}{\sqrt{a^{2}-1}} .
$$

As seen from Eq.(13), the current-phase relation has two components, $\sin \varphi$ and $\sin \varphi / 2$. The $\operatorname{sign}$ of the $\sin \varphi / 2$ component is determined by the sign of the interlayer pair potential $\Delta_{s^{\prime}}$, which is determined selfconsistently from Eq.(14) and depends on the sign of the electron-electron interaction in an $S^{\prime}$ material. For an attractive interaction $\left(T_{c s^{\prime}}>0\right) \Delta_{s^{\prime}}$ does not vanish even at $T>T_{c s^{\prime}}$ and has a positive sign, while for a repulsive interaction $\Delta_{s^{\prime}}$ becomes negative. Therefore the measurements of a current-phase relation in a $S I S^{\prime} I S$ junction can be used for measuring the sign of the electronelectron interaction in metallic films.

As is shown above, the critical current is controlled by a single suppression parameter $\gamma_{\text {eff }}=\pi T_{c} / \Gamma=$ $2 \pi T_{c s} d / \hbar v_{F}\langle x D(x)\rangle$. As follows from Eq.(13), for $T>$ $T_{c s^{\prime}}$ and $\gamma_{e f f}\left(\omega_{n} / \pi T_{c s}\right) \gg 1$ the supercurrent becomes of the order of $\langle x D(x)\rangle^{2}$ as expected for the incoherent tunneling in a double-barrier contact:

$$
e J_{s} R_{N}=\frac{32 \pi T}{7 \gamma_{e f f}} \sum_{\omega_{n}} \frac{\Delta_{s}^{2} \sin \varphi}{E_{1}^{2}} .
$$

A numerical evaluation of Eq.(13) is shown in Fig. 1. The coherent (I) and incoherent (II) regimes are indicated. Regime number III shows the crossover to the series connection of two SIS' tunnel junctions.

Transmission distribution, MAR. The result for the coherent regime Eq.(11) can be derived from the transmission eigenvalue density $\rho(D)=\left(G_{N} / \tau_{-} G_{0}\right) D^{-3 / 2}(1-$ $D)^{-1 / 2}$ for two-barrier NININ contactst, where $G_{0}=$ $e^{2} / 2 \pi \hbar$. While deriving this distribution, an assumption about a certain amount of impurity scattering in the interlayer was madet; it can be shown that it also holds for the considered case of a clean interlayer, provided that $k_{F} d \gg 1$.

The derivation of Eq.(11) is then performed by the calculation of the integral $\int_{0}^{1} I_{c}(D) \rho(D) d D$, where $I_{c}(D)$ is a supercurrent per single ballistic channel, $I_{c}(D)=(e / 2 \hbar) \Delta_{s}^{2} D \sin \varphi \tanh \left(E_{B} / 2 T\right) / E_{B}$ and $E_{B}=$ $\Delta \sqrt{1-D \sin ^{2} \varphi / 2}$, by the residues of $\tanh (x)$ in a complex plane $z=(1 / D-1)^{1 / 2}$. It yields exactly the coherent supercurrent $e J_{s} R_{N}=2 \pi T \sum_{\omega>0} \Delta_{s}^{2} \sin \varphi / E_{1} E_{3}$. This proof also holds for the asymmetric case, when the eigenvalue density is $\rho(D)=\left(G_{N} / \pi G_{0}\right) D^{-3 / 2}\left(D_{\max }-D\right)^{-1 / 2}$ with $D_{\max }=4 \gamma_{1} \gamma_{2} /\left(\gamma_{1}+\gamma_{2}\right)^{2}, 0<D<D_{\max }$.

The considerations above allow one to calculate the current under finite voltage $V$ at the contact on the basis of the MAR (multiple Andreev reflections) 


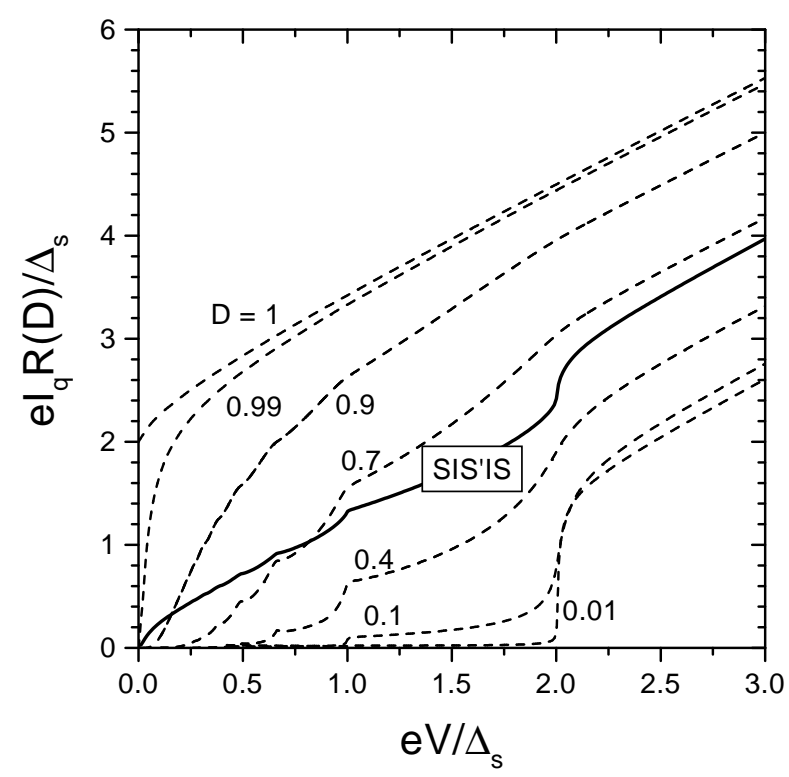

FIG. 2. Dc current component in $S I S^{\prime} I S$ contact in the coherent regime, together with the single-channel curves for various transmissions $\mathrm{D}$.

formalism 18 21. Below we discuss the dc current component in a symmetric $S I S^{\prime} I S$ contact. The dc current per single channel with transmisison $\mathrm{D}$ is given by 20

$$
\begin{aligned}
\pi \hbar I_{q}(D, V) / e= & e V D-\int d \varepsilon \tanh \frac{\varepsilon}{2 T}\left(1-\left|a_{0}\right|^{2}\right) \\
& \times\left(2 \operatorname{Re}\left(a_{0} A_{0}\right)+\sum_{n}\left(\left|A_{n}\right|^{2}-\left|B_{n}\right|^{2}\right)\right),
\end{aligned}
$$

where $a_{n}=a(\varepsilon+n e V)$ is the Andreev reflection amplitude: $a(\varepsilon)=\left(\varepsilon-\operatorname{sgn}(\varepsilon)\left(\varepsilon^{2}-\Delta^{2}\right)^{1 / 2}\right)$ for $|\varepsilon|>\Delta$ and $a(\varepsilon)=\left(\varepsilon-i\left(\Delta^{2}-\varepsilon^{2}\right)^{1 / 2}\right)$ for $|\varepsilon|<\Delta$. The coefficients $A_{n}, B_{n}$ are given by the recurrency relations which were derived in 20 and can easily be solved numerically.

The resulting total dc current in a symmetric $S I S^{\prime} I S$ contact is given by the integration of the singlechannel result $I_{q}(D, V)$ over the eigenvalue density $I_{q}=$ $\int_{0}^{1} I_{q}(D, V) \rho(D) d D$ where $\rho(D)=\left(G_{N} / \pi G_{0}\right) D^{-3 / 2}(1-$ $D)^{-1 / 2}$.

The results of numerical calculation at temperatures $T \ll T_{c s}$ are presented in Fig.2. A few single-channel curves for various $\mathrm{D}$ are also shown for comparison. The excess current $I_{e x} e R_{N} \simeq 1.05 \Delta_{s}$ is present at high bias $\mathrm{eV} \gg \Delta_{s}$, while the subharmonic gap structure at $e V=2 \Delta_{s} / n$ due to MAR is present at lower voltages, despite averaging over the channels. This dc current determines the amount of dissipation in the junction. Note that the curve $S I S^{\prime} I S$ in Fig.2 is universal, i.e. independent of microscopic parameters such as electronic mean free path or $T_{c}$ of the interlayer as long as $\gamma_{e f f}<1$. This universality breaks down with the increase of $\gamma_{e f f}$, due to the dephasing of the transmission resonances. The de- tailed theory applicable for arbitrary $\gamma_{e f f}$ is the subject of further study.

In conclusion, the general solution for the supercurrent in double-barrier Josephson junctions is presented and the crossover from the coherent to the incoherent regime for increasing interlayer thickness is studied in detail. The parameter-free calculation of quasiparticle current is done in the coherent regime. The results have implications for transport in Josephson junctions and multilayers engineered with modern techniques.

The authors thank M.Yu. Kupriyanov, K.K. Likharev, Yu.V. Nazarov, I.P. Nevirkovets and H. Rogalla for many useful and stimulating discussions.

${ }^{1}$ C.W.J. Beenakker, Rev. Mod. Phys. 69, 731 (1997).

${ }^{2}$ G.E. Blonder, M. Tinkham, and T.M. Klapwijk, Phys. Rev. B 25, 4515 (1982).

${ }^{3}$ A.F. Volkov, A.V. Zaitsev, and T.M. Klapwijk, Physica C 210, 21 (1993).

${ }^{4}$ Yu.V. Nazarov, Phys. Rev. Lett. 73, 1420 (1994).

${ }^{5}$ J.A. Melsen and C.W.J. Beenakker, Physica B 203, 219 (1994).

${ }^{6}$ B.D. Josephson, Phys. Lett. 1, 251 (1962).

${ }^{7}$ K.K. Likharev, Rev. Mod. Phys. 51, 101 (1979).

${ }^{8}$ I.P. Nevirkovets, J.B. Ketterson, and S. Lomatch, Appl. Phys. Lett. 74, 1624 (1999).

${ }^{9}$ H.Schulze, R.Behr, F.Müller, and J.Niemeyer, Appl. Phys. Lett. 73, 996 (1998).

${ }^{10}$ M.Yu. Kupriyanov and V.F. Lukichev, Sov.Phys. JETP 67, 1163 (1988).

${ }^{11}$ A.L. Gudkov, M.Yu. Kupriyanov, and K.K. Likharev, Sov. Phys. JETP 68, 1478 (1988).

12 A. Furusaki, H. Takayanagi, and M. Tsukada, Phys. Rev. B 45, 10563 (1992).

${ }^{13}$ A. Chrestin, T. Matsuyama, and U. Merkt, Phys. Rev. B 49, 498 (1994).

${ }^{14}$ G. Johansson, E.N. Bratus', V.S. Shumeiko, and G. Wendin, Phys. Rev. B 60, 1382 (1999).

${ }^{15}$ D.V. Goncharov, I.A. Devyatov, and M.Yu. Kupriyanov, in Proceedings of Nanostructures, Physics and Technology, 1998 (St.Petersburg, Russia), p. 241-244.

${ }^{16}$ I.P. Nevirkovets and S.E. Shafranjuk, Phys. Rev. B 59, 1311 (1999).

17 M.J.M. de Jong, Phys. Rev. B 54, 8144 (1996).

18 E.N. Bratus', V.S. Shumeiko, and G. Wendin, Phys. Rev. Lett. 74, 2110 (1995).

${ }^{19}$ D.V. Averin and A. Bardas, Phys. Rev. Lett. 75, 1831 (1995).

${ }^{20}$ A. Bardas and D.V. Averin, Phys. Rev. B 56, R8518 (1997).

21 J.C. Cuevas, A. Martin-Rodero, and A. Levy Yeyati, Phys. Rev. B 54, 7366 (1996). 